\title{
A plethysmographic sensor for monitoring volume changes in cardiovascular pathologies
}

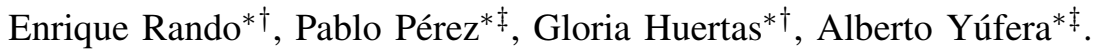 \\ ${ }^{*}$ Instituto de Microelectrónica de Sevilla, IMSE, CNM (Universidad de Sevilla, CSIC) \\ ${ }^{\dagger}$ Departamento de Electrónica y Electromagnetismo, Facultad de Física, Universidad de Sevilla \\ ‡Departamento de Tecnología Electrónica, Escuela Técnica Superior de Ingeniería Informática, Universidad de Sevilla.
}

\begin{abstract}
This paper presents a capacitive system capable of performing leg volume change monitorization in patients suffering from Heart Failure (HF) conditions. The body volume evolution serves as a prognosis marker for this kind of patients, such patients can benefit from a wearable monitorization system. The proposal is based on a contactless capacitive wearable structure implemented by a two-plate plane-parallel capacitor geometry. The system exhibits sensitivity to leg volume change and the sensor curves are provided. A $2.5 \times 2.5 \mathrm{~cm}$ capacitive electrode design will generate capacitive values within the range [1-2] pF. Acquisition of the capacitance value is performed via an electronic differentiator implemented using op-amps, illustrating good results in simulated volume analysis implemented using pspice.
\end{abstract}

\section{INTRODUCTION}

Heart failure (HF) is a clinical consequence of many heart diseases. It is diagnosed by several symptoms and signs (e.g., dyspnea, swelling of the legs) that result from abnormalities in cardiac structure and/or function [1]. Cardiac pump failure may cause an overload of liquids with the subsequent accumulation of pulmonary and systemic liquids. The acute decompensated heart failure (DHF) is often a potentially lifethreatening condition requiring hospitalization and emergency treatments, which involve managing the liquid excess [1]. The acute $\mathrm{HF}$ is a common and costly disease. In most European countries, the acute heart failure is the main cause of hospitalization in people over 65 , and is responsible for $1-2 \%$ of all medical care expenses, mainly due to the hospitalization costs [2]. Results for heart failure are worse than breast, prostate and intestine cancer [3]. The body volume excess (liquid overload) due to the neuro-hormonal activation is the main factor leading to $\mathrm{HF}$ hospitalizations [1]. The standard evaluation of $\mathrm{HF}$ (signs and symptoms, image tests and natriuretic peptides analysis) does not reliably predict DHF or clinical outcomes after hospitalizations.

The electrical impedance of the biological material, commonly known as Bioimpedance or BI, has been widely used for the characterization of cells [4], tissues and organs [5], among others, since the biological electrical properties (permittivity and conductivity) are closely related to the physical physiological state of biological matter. Due to this, BI is an excellent potential bio-marker for medical diagnosis [6], [7]. These electrical properties depend on frequency, which allows spectroscopy techniques to be widely used to test BI (Electrical Impedance Spectroscopy, EIS). In addition, BI tests are non- invasive, inexpensive, and easy to implement. BI-based studies have reported estimates of some parts (mainly intrathoracic) or the volume of the entire body [6], [8]. These studies are based on the inverse relationship between body impedance and volume [6], which allows BI systems to estimate the volume of interest by placing electrodes on the corresponding body part.

The devices developed for BI tests are implemented using relatively complex electronic systems, generally based on commercial equipment that lead to non-portable and heavy devices. There are several example of BI devices on the market, however, these devices are not useful for real-time continuous BI monitoring in daily clinical practice, e.g. Medtronics (Optivol), RJL Systems (Quantum/S Analyzer). Some approaches have been implemented for portable devices [6] but they are not comfortable enough for continuous monitoring of BI, and should be optimized for low power consumption and wireless connection. In acute heart failure, BI volume measurements can serve as a potential bio-marker for diagnosis, in [9] BI analysis is employed to calculate the edema index as the relationship between extracellular body water and total body water. BI analysis illustrate potential as a bio-marker for HF, in [10] leg impedance is identified as the most relevant parameter. However, fluids in humans follow a dynamic process with daily circadian changes and, in patients with heart failure, may depend on the stage, degree of (de)compensation and treatment; the dynamics of fluid overload in patients with acute heart failure during hospitalization and after discharge have not been previously described. The objective of this study is to provide a BI measurement technique oriented towards the development of wearable body fluid sensor, capable of performing volume change monitorization, to analyze leg fluid accumulation.

This article proposes an alternative and new method for the evaluation of body volume, based on capacitive sensing performed by a plane-parallel structure of capacitors at $180^{\circ}$ located on the ankle (Figure 1). In section II, the model for the proposed capacitive sensor is described, together with a first circuit option for electrical test. In section III finite element analysis of the sensor and electrical simulations of the measurement circuit are displayed using real experimental data [12]. A test example is also described for a venousocclusion plethysmography assay. Finally, some conclusions are described in section IV. 


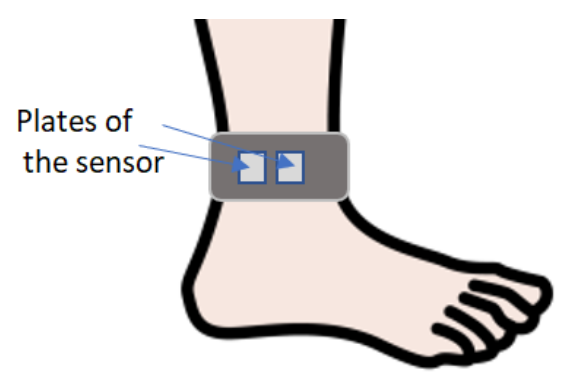

Fig. 1. Placement of the proposed sensor in the patient's leg.

\section{MATERIALS AND METHODS}

\section{A. Sensor model}

The proposed sensor is formed by two squared plates at a $180^{\circ}$ angle, implementing a capacitor, placed at a certain distance of the leg. When the leg swells or deflates, the capacitance will vary accordingly, related to the leg position with respect to the plates. The volume variation can be obtained by performing periodical acquisition of the sensor capacitance.

The sensor model can be analytically described from the physical characteristic of the designed device. Assume a parallel capacitor formed by two squared plates with length and width respectively denoted as $l_{0}$ and $t_{0}$. The plates are equally spaced at a distance $d_{0}$.

If a rectangular parallelepiped dielectric of dimensions $l_{1}$. $t_{0} \cdot d_{1}$ and relative permittivity $\epsilon_{r}$ is placed betweeen the plates (fig. 2), the capacitance is given by two parallel capacitors in the following form: A capacitor of surface $t_{0} \cdot\left(l_{0}-l_{1}\right)$ and length $d_{0}$ with permittivity $\epsilon_{0}$, which capacitance is:

$$
C_{1}=\frac{\epsilon_{0} \cdot\left(l_{0}-l_{1}\right) \cdot t_{0}}{d_{0}}
$$

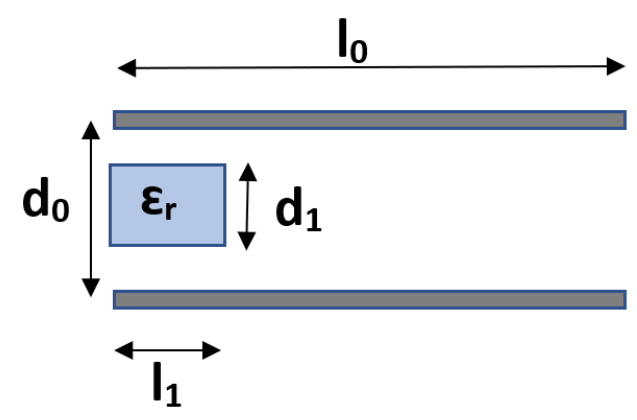

Fig. 2. Preliminary model used to extrapolate results to the actual problem. The width of the electrodes and the dielectric is $t_{0}$.

Two capacitors in series arise from the dielectric introduced, both of surface $l_{1} \cdot t_{0}$, one of length $d_{1}$ and relative permittivity $\epsilon_{r}$ and another of length $d_{0}-d_{1}$ and permittivity $\epsilon_{0}$ :

$$
\begin{gathered}
C_{21}=\frac{\epsilon_{0} \cdot \epsilon_{r} \cdot l_{1} \cdot t_{0}}{d_{1}} \\
C_{22}=\frac{\epsilon_{0} \cdot l_{1} \cdot t_{0}}{d_{0}-d_{1}}
\end{gathered}
$$

$$
C_{2}=\left(\frac{1}{C_{21}}+\frac{1}{C_{22}}\right)^{-1}=\frac{\epsilon_{0} \cdot l_{1} \cdot t_{0} \cdot \epsilon_{r}}{d_{0} \cdot \epsilon_{r}+d_{1} \cdot\left(1-\epsilon_{r}\right)}
$$

Then, the capacity of the whole capacitor is:

$$
C=C_{1}+C_{2}=\frac{\epsilon_{0} \cdot\left(l_{0}-l_{1}\right) \cdot t_{0}}{d_{0}}+\frac{\epsilon_{0} \cdot l_{1} \cdot t_{0} \cdot \epsilon_{r}}{d_{0} \cdot \epsilon_{r}+d_{1} \cdot\left(1-\epsilon_{r}\right)}
$$

According to the literature, human body parts (skin, blood and muscle) have a $\epsilon_{r}$, at $10 \mathrm{KHz}$ (the operation frequency of the measurement circuit), at least higher than 100 [11], so the approximation $1-\epsilon_{r} \simeq-\epsilon_{r}$ can be made (if $d_{0} \neq d_{1}$ ) and the first term can be neglected when $l_{0}$ and $l_{1}$ have comparable values:

$$
C \simeq \epsilon_{0} \cdot t_{0} \cdot \frac{l_{1}}{d_{0}-d_{1}}
$$

Notice the relation between the capacitance and the dielectric, depending only on the dielectric size and not on the relative permittivity.

Recalling our initial definition for a wearable sensor around the leg the assumption that sensor capacitance is equivalent to (6) is proposed. However, for this specific application, $l_{1}, d_{0}$ and $d_{1}$ need to be redefined (figure 3 ).

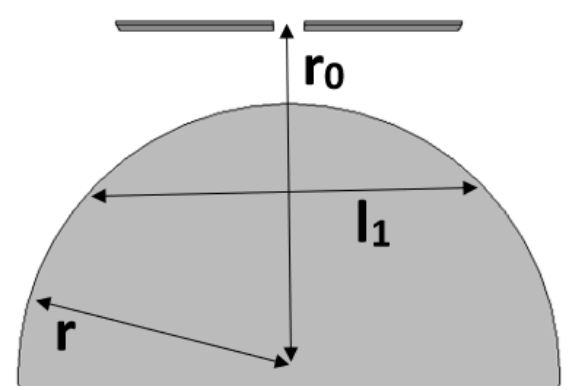

Fig. 3. Schematics of the final sensor model and the leg in cross section.

The geometry of the leg can be simplified to a cylinder, which radius is $r$ and its length is much longer than the plates of the sensor. There is a distance $r_{0}$ from the center of the cylinder to the plates. $d_{0}-d_{1}$ which correspond in the previous simplified example to the distance not occupied by the rectangular dielectric between the parallel plate capacitor, can now be estimated as $r_{0}-r$, which equivalently is the distance not occupied by the cylindrical dielectric between the sensor and the center of the cylinder.

Establishing a relation between $l_{1}$ and $r$ is not as simple. $l_{1}$ is the length of the dielectric perpendicular to $d_{1}$, but in the case of the cylinder this varies with the distance from the sensor to the point where $l_{1}$ is to be calculated. Averaging $l_{1}$ is not an accurate solution, as the contribution of it to the capacitance is much higher at close distances to the sensor, but at further distances it is negligible. This value can be approximated by applying the following constraints:

- There is symmetry in the most of $t_{0}$, so the problem can be reduced to two dimensions.

- The surface is enclosed within a semi-circumference which radius is half the distance between the electrodes 
plus the length of them $\left(\rho_{\max }\right)$ is the one that mainly contributes to the capacitance, as the electric field is much more intense in that region. Then, the study can be reduced to only that surface and, to simplify, border effects are neglected. The area limited by a circumference whose radius is half the distance between the electrodes $\left(\rho_{\min }\right)$ will also be excluded to simplify the calculations (figure 4).

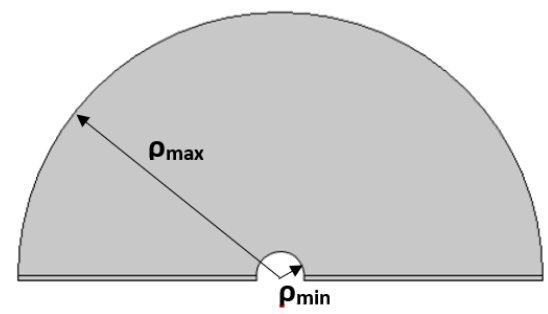

Fig. 4. Schematics of the the surface of study in an approximated twodimensional case.

The electric potential has radial symmetry, so it can be calculated with the laplacian in polar coordinates:

$$
V(\phi)=\frac{V_{0}}{\pi} \cdot \phi
$$

where $V_{0}$ is the electric potential between the electrodes.

The electric field can be calculated by $\vec{E}=-\vec{\nabla} V$ in polar coordinates:

$$
E(\rho)=\frac{V_{0}}{\pi \cdot \rho}
$$

Being $\rho$ the distance from the electrodes to any point in the region of study.

- Since the leg has a very high permittivity, the electric field inside can be neglected. Then, the expression for the potential is:

$$
V(\rho, \phi)=\frac{V_{0} / 2}{\phi_{\text {cut }} \cdot(\rho)} \phi, \quad \phi \in\left[0, \phi_{\text {cut }}(\rho)\right)
$$

where $\phi_{c u t}$ is the angle where the leg and the air are coincident for a certain $\rho$.

Only the field in the electrode is useful, as it is what it is needed to calculate the charge. As the plate is conductive, the field must be perpendicular to the surface there:

$$
E(\rho)=\frac{V_{0} / 2}{\phi_{\text {cut }}(\rho) \cdot \rho}
$$

- The electric field has to be continous in the plate.

For $\rho>r_{0}-r$, (9) can be used. For a lower $\rho$ this expression makes no sense, as the electric field lines do not cross through the leg. Therefore, (8) can be used with a constant $\mathrm{K}$ that allows the field to be continuous.

$$
\begin{array}{cc}
E(\rho)=\frac{V_{0}}{\pi \cdot \rho}+K(\rho), & \rho \in\left[\rho_{\text {min }}, r_{0}-r\right) \\
E(\rho)=\frac{V_{0} / 2}{\phi_{\text {cut }}(\rho) \cdot \rho}, & \rho \in\left[r_{0}-r, \rho_{\max }\right)
\end{array}
$$

Once this calculations are performed and the electric field is obtained, the charge density can be calculated with $\sigma(\rho)=$ $\epsilon_{0} \cdot E(\rho)$ and integrated respect to the variable $\rho$ obtaining the charge per unit length. Then, dividing that charge by $V_{0}$ generates the capacitance per unit length value measured by the sensor. The relation between the capacitance and the capacitance per unit length with the radius of the leg must be the same since the system is assumed to have symmetry in the direction of $t_{0}$.

In section III.A, the formula that relates the capacitance of the sensor with the radius of the leg will be obtained, in both $2 \mathrm{D}$ and $3 \mathrm{D}$ cases.

\section{B. Measurement circuit: Op amp Differentiator.}

The circuit must be able to measure the capacitance of the sensor with the required accuracy in the picofarads range. Additionally, the sensor must be able to monitor changes in the capacitance over time. The result must be accurate and simple to implement, yet able to work at $9 \mathrm{~V}$ or lower, and at a frequency that can be produced by a standard microcontroller and that does not decrease the relative permittivity to less than 100 [11], that is according to the scientific literature, at $10 \mathrm{kHz}$.

Taking into consideration all these factors, a differentiator circuit has been chosen for its simplicity and high accuracy (figure 5).

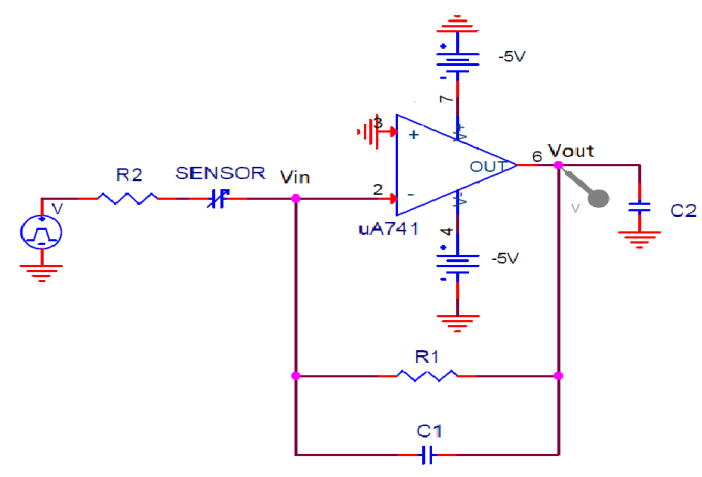

Fig. 5. Schematics of the circuit designed to measure the capacitance of the sensor

The input voltage is a triangular signal of $10 \mathrm{~V}$ peak-topeak, with a period of $0.1 \mathrm{~ms}$. R1 is the sensing resistance, of $2.5 \mathrm{M} \Omega$. Then, the capacitance of the sensor $\left(C_{\text {sensor }}\right)$ is

$$
C_{\text {sensor }}=-\frac{V_{\text {out }}}{m \cdot R}=-2 \cdot V_{\text {out }} \mathrm{pF} / \mathrm{V},
$$

where $m$ is the slope of the input voltage. An example of this measurement can be seen in figure $6 . V_{\text {out }}$ is the derivative of the triangular input signal. The $V_{\text {out }}$ amplitude value is related to the sensor capacitance value.

Resistance R2 $(30 \mathrm{k} \Omega)$ and capacitor $\mathrm{C} 2(1 \mathrm{pF})$ are used in order to create a low pass filter that attenuates the high frequency noise, as the differentiator circuit only by itself would amplify it.

The operational amplifier used for the circuit is a UA741. It is fed by 5 and $-5 \mathrm{~V}$ voltage sources, allowing it to measure 


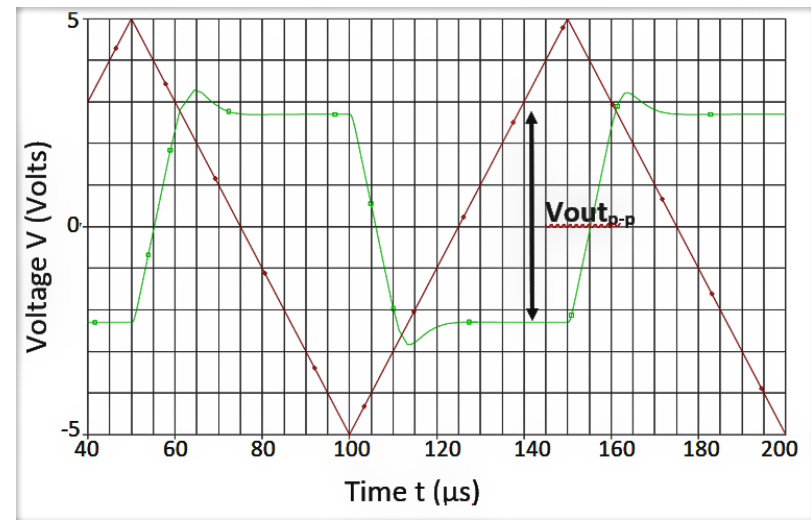

Fig. 6. Plot of input signal (red) and output signal (green) of the sensing circuit, as peak to peak value is $5 \mathrm{~V}$. Output signal is the differentiated of the input, Then, applying (13), capacitance is $5 \mathrm{pF}$.

capacitance values up to $10 \mathrm{pF}$. As the resistance $\mathrm{R} 1$ has a high value for this operational amplifier, a little amount of current will go from the positive input to the negative input, causing some offset in the output signal. However, this can be easily solved by measuring peak-to-peak value instead of the absolute magnitude.

\section{RESULTS}

\section{A. Simulation of the capacitive sensor in COMSOL}

The two-dimensional problem depicted on previous section is simulated to compare its results with the analytic approximation solutions of (11) and (12). The leg (cylinder) is placed at $5.5 \mathrm{~cm}$ from the sensor, whose plates are separated by 0.5 $\mathrm{cm}$ and their length is $2.5 \mathrm{~cm}$. The voltage between the plates $\left(V_{0}\right)$ is $1 \mathrm{~V}$ and the relative permittivity of the leg is $\epsilon_{r}=300$.

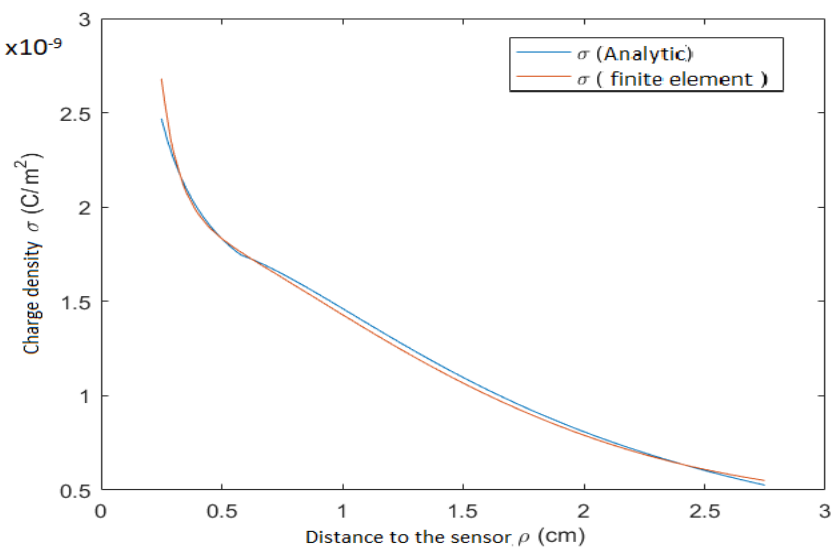

Fig. 7. Plot of the charge density in the plates respect to the distance from the center of the sensor. The radius of the leg is $5.25 \mathrm{~cm}$. The orange curve is obtained by finite element simulation and the blue one by the equations (11) and (12) (analytic).

Charge density $\sigma(\rho)$ is represented for a radius of the leg $r=5.25 \mathrm{~cm}$ in figure 7 . Both simulation and analytical results are very similar. If this curve is integrated and divided by $V_{0}$, the capacitance of the sensor can be obtained. Performing this calculation for different radii permits to plot the radius of the leg versus capacitance curve (figure 8).

$$
C=A \cdot \frac{r^{2}}{r_{0}-r}+B \mathrm{pF}
$$

where $A$ is $(0.231 \pm 0.001) \mathrm{pF} /$ cencmtimeters, $r_{0}$ is $(5.576 \pm 0.002) \mathrm{cm}$ and $B$ is $(6.56 \pm 0.02) \mathrm{pF} . r^{2}$ is 0.9999 .

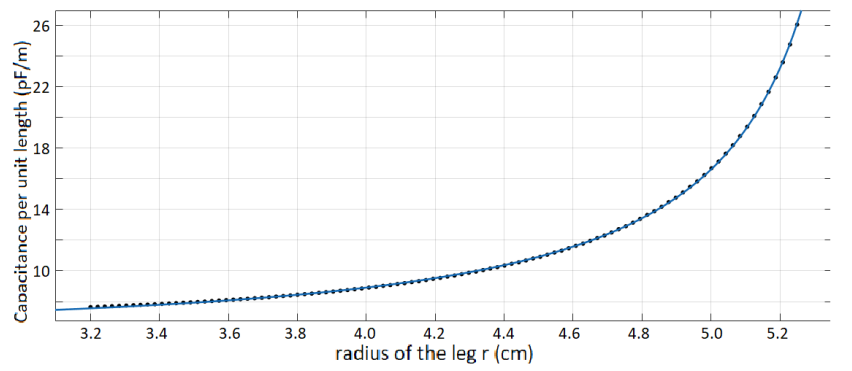

Fig. 8. Capacitance of the sensor per unit length versus radius of the leg. Two-dimensional case. Regression curve $A \frac{r^{2}}{r_{0}-r}+B$ vs r.

Second, the three-dimensional case is analyzed and simulated to validate the expression (14). In this case, this time without approximations or restrictions. The relation between the capacitance and the radius is expected to be the same, since there is symmetry in the direction of $t_{0}$.

The leg (cylinder) is placed at $5.5 \mathrm{~cm}$ from the sensor, whose plates are separated by $0.5 \mathrm{~cm}$ and their length and width are $2.5 \mathrm{~cm}$. The voltage between the plates $\left(V_{0}\right)$ is $1 \mathrm{~V}$. The length of the cylinder does not vary the result as long as it is higher than $6 \mathrm{~cm}$ and its relative permittivity is $\epsilon_{r}=300$. Changes in the relative permittivity does not affect in the, as long as it is higher than approximately 200 .

The simulation is made for a range of radii, in order to plot the curve radius of the leg versus capacitance (figure 9).

This curve is also adjusted following (14) (figure 9): where $A$ is $(0.0045 \pm 0.0002) \mathrm{pF} / \mathrm{cm}, r_{0}$ is $(5.536 \pm 0.002) \mathrm{cm}$ and $B$ is $(0.404 \pm 0.006) \mathrm{pF} . r^{2}$ is 0.999 .

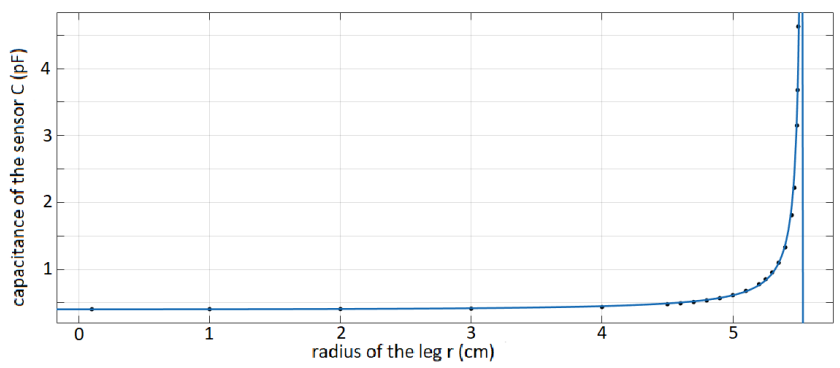

Fig. 9. Capacitance of the sensor versus radius of the leg. Three-dimensional case. Regression curve $A \frac{r^{2}}{r_{0}-r}+B$ vs r.

The relation between the radius and the capacitance remains the same. 


\section{B. Circuit Simulation in PSPICE}

The circuit to be simulated is illustrated in figure 5. For the sensor capacitance, (??) is used for radii from 0 to $5.5 \mathrm{~cm}$ in order to obtain its capacitance. Using (13), the capacitance is calculated from the output signal of the simulation, which is plotted in figure 10. It is almost identical to the one obtained by finite element simulation (figure 9). The relative error of the measurement is, in the worst case scenario, $0.5 \%$ (figure 11).

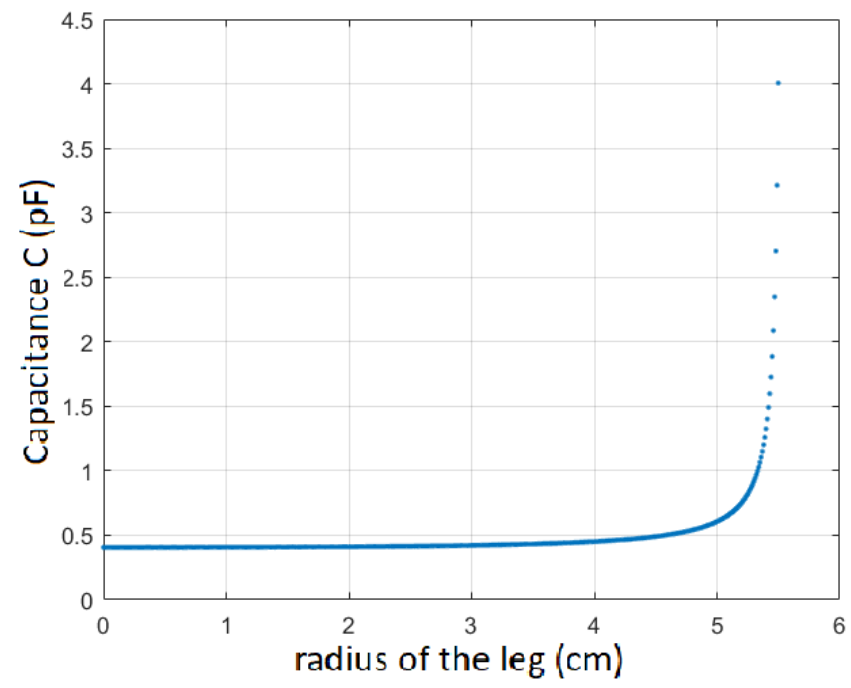

Fig. 10. Capacitance measured in the PSPICE simulation versus the radius of the leg.

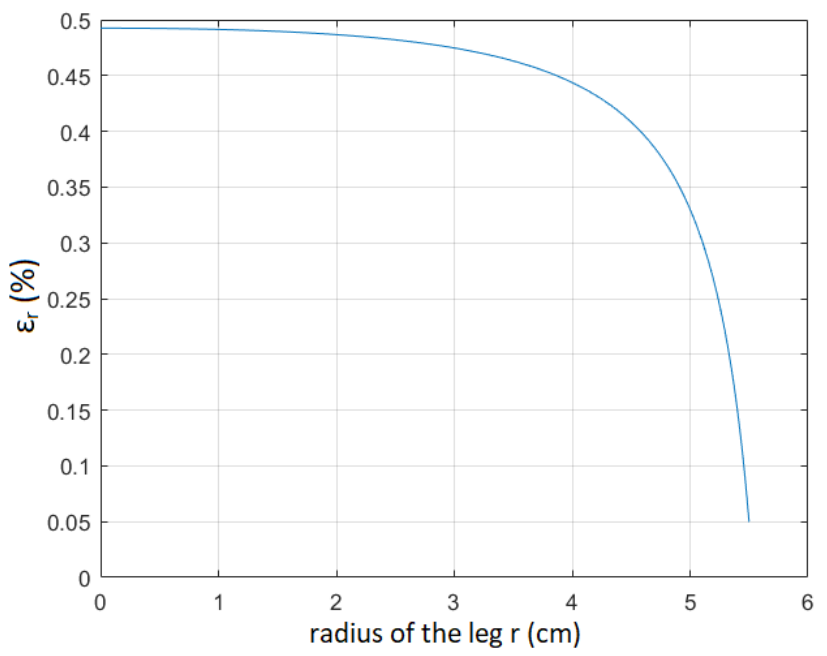

Fig. 11. Relative error of the capacitance obtained by the simulation and the fitted curve (??) versus the radius of the leg.

C. Simulation of the circuit using venous-occlusion plethysmography data

In venous-occlusion plethysmography, a venous-occlusion cuff is inflated in order to stop venous return. Arterial flow causes an increase in volume of the leg segment, which is attempted to be measured with the capacitive sensor.

According to [12] the volume increase curve in the experiment, follows the curve represented in blue in figure 12. In order to evaluate the proposed sensor and circuit and test the potential to perform these measurements, this experiment is simulated.

The leg, modelled as a cylinder, has an initial radius of $5.3 \mathrm{~cm}$ (a typical value). The change of volume is considered to take place in $3 \mathrm{~cm}$ of the leg. With this considerations, the curve radius versus time is calculated, and is converted to capacitance versus time with (14). A time-varying capacitance is simulated in the circuit shown in figure 5 for 30 seconds.

Once the simulation is completed, the curve capacitance versus time is calculated from the voltage peak-to-peak of the output signal using (13) thus the inverse process is performed, obtaining volume difference versus time from the simulation (figure 12).

The results show that the volume is successfully determined over time, with a relative error lesser than $1 \%$, which is mainly due to the tolerance of the simulation.

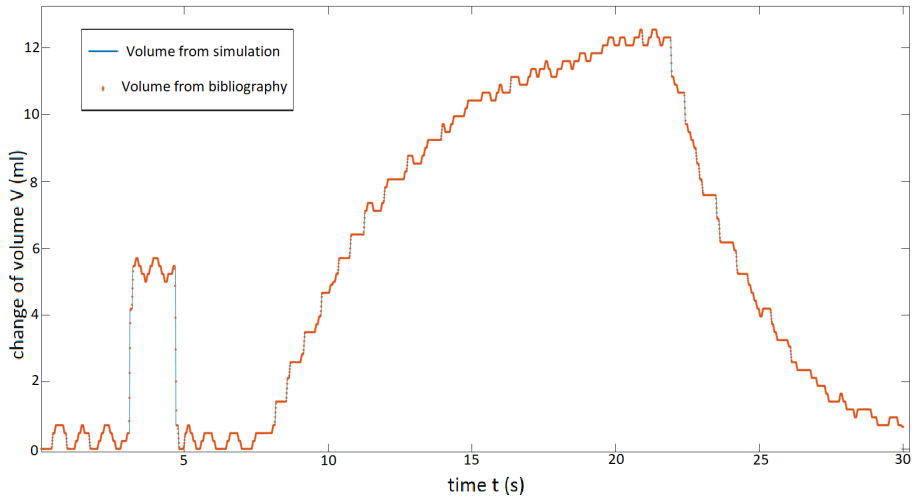

Fig. 12. Volume increase from measured capacitance in the PSPICE simulation and volume increase from bibliography versus time.

\section{CONCLUSION}

This paper describes a wearable prototype proposal including a capacitive sensor useful for real-time status monitoring of heart failure patients, based on the leg volume measurement. A two plate capacitive sensor, obtaining the sensitivity curves valid for leg volume test has been proposed analyzing the capacity range to be measure. As the measurement is local and done from a close distance, approximating the leg to a cylinder is valid. The sensor has been positively tested in electrical simulations with a diferentiator circuit. In the future, this idea could be implemented in a fully-integrated and wearable sensor for heart failure patients supervision.

\section{ACKNOWLEDGMENT}

The work has been supported by Monitorizacin en Tiempo Real de Variables Hemodinmicas con Stents Inteligentes (Istent) Mediante Sensores Capacitivos y Bioimpedancia. (RTI2018-093512-B-C21) 
We would like to thank to Dr. Javier Medrano Ortega, from the Instituto de Biomedicina de Sevilla (IBIS), for its valuable advice and medical supervision.

\section{REFERENCES}

[1] P. Ponikowskietal. Guidelines for the diagnosis and treatment of acute and chronic heart failure. Eur Heart J., 37, 2129 -200, 2016.

[2] A. Auricchio F. Braunschweig F, M.R. Cowie. What are the costs of heart failure? Europace, 13 Suppl 2, ii13-ii17, 2011.

[3] S. Stewart S et al. More malignant than cancer? Five-year survival following a first admission for heart failure. Eur J Heart Fail, 3, 315-22, 2001.

[4] P. Perez et al.Sensing Cell-Culture Assays with Low-Cost Circuitry. Sci Rep. (Nature group), 8, 8841, 2018.

[5] A. Yufera et al. A Tissue Impedance Measurement Chip for Myocardial Ischemia Detection. IEEE TCASI, 12, 2620-2628, 2005.

[6] S. Lee et al. Congestive Heart Failure Patient Monitoring usingWearable Bio-impedance Sensor Technology. Conf Proc IEEE EMBS, 8, 438-41, 2015.

[7] S. Grimnes \& O. Martinsen. Bioimpedance and Bioelectricity Basics. Elsevier, 2014.

[8] E. Pitella et al. Metrological characterization of a combined bioimpedance plethysmograph and spectrometer. Elsevier, Measurement, 120, 221-29, 2018.

[9] K.J. Lyons et al. Noninvasive Bioelectrical Impedance for Predicting Clinical Outcomes in Outpatients With HF. Crit Pathw Cardiol 16, 32-36, 2017.

[10] D. Lindholm et al. Bioimpedance and New-Onset HF: A Longitudinal Study of 500000 Individuals. J Am Heart Assoc., 7, pii: e008970, 2018.

[11] Camelia Gabriel, The Dielectric Properties of Biological Materials. Radiofrequency Radiation Standards, pp. 187-196, 2013.

[12] John G. Webster (Editor), Medical Documentation. Instrumentation and Design, 4th ed. pp. 364-366, 2009. 